\title{
Estudio Estadístico De Lacaracterización De Residuos Solidos De La Parroquia Taracoa Provincia De Orellana
}

\author{
Juan Pablo Wayllas Pazmiño (Ing., Amb., MSc.) \\ Universidad/Escuela Superior Politécnica de Chimborazo \\ Extensión Norte Amazónica de la Republica de Ecuador
}

Doi: 10.19044/esj.2018.v14n2p304 URL:http://dx.doi.org/10.19044/esj.2018.v14n2p304

\begin{abstract}
In this study, a statistical analysis was carried out on the management of solid household waste generated in the Taracoa parish of the province of Orellana in the Ecuadorian Oriente. The inhabitants of the study community must, however, observe the legal regulations provided by the municipality of Orellana. The analysis was aimed at identifying the per capita production of solid waste and the characterization of them. 61 families were chosen to carry out the study, with an average of 5 inhabitants for each of them, resulting in a total of 305 inhabitants. The methodology of this study uses the probabilistic method to obtain the samples. These samples were taken directly from the houses identified for sampling. The results show that the solid waste PPC in the parish Taracoa of the province of Orellana was $0.89 \mathrm{~kg} / \mathrm{hab} / \mathrm{day}$, generating a total of $273.60 \mathrm{~kg} /$ day. This, therefore, was sent to the dump of the municipality of Orellana. The statistical analysis determines that in the domiciles of the parish Taracoa of the province of Orellana, organic waste occupies the first place with $75.60 \%$. This was followed by plastic sleeves with $8.60 \%$, plastic bottles with $5.20 \%$, carton with $4.90 \%$, paper with $3.95 \%$, and cans with $1.75 \%$.
\end{abstract}

Keywords: Study, management, waste, Taracoa, Orellana

\section{Resumen}

En este estudio se realizó un análisis estadístico sobre el manejo de los residuos sólidos domiciliarios generados en la parroquia Taracoa de la provincia de Orellana en el oriente ecuatoriano, los habitantes de la comunidad en estudio deben observar la normativa legal que dispone la municipalidad de Orellana. El análisis realizado tuvo el objetivo de identificar la producción per cápita de desechos sólidos, y la caracterización de los mismos. Se escogieron 61 familias para realizar el estudio, con un promedio de 5 habitantes por cada 
una de ellas arrojando un total de 305 habitantes. La metodología utilizada fue utilizar el método probabilístico para obtener la muestra, estas se tomaron directamente de las viviendas identificadas para el muestreo. Se evidenciaron los siguientes resultados; la PPC de desechos sólidos en la parroquia Taracoa de la provincia de Orellana fue de $0,89 \mathrm{~kg} / \mathrm{hab} /$ díagenerando un total de 273,60 $\mathrm{kg} /$ día, los cuales son enviados al botadero del Municipio de Orellana.El análisis estadístico determina que en los domicilios de la parroquia Taracoa de la Provincia de Orellana los desechos orgánicos ocupan el primer lugar con el $75,60 \%$, seguido de las fundas plásticas con el $8,60 \%$, los botellas de plástico con el 5,20\%, el cartón con el 4,90\%, el papel con el 3,95\% y latas con el $1,75 \%$.

Palabras clave: Estudio, manejo, residuos, Taracoa, Orellana

\section{INTRODUCCIÓN}

La generación de residuos sólidos es inherente a la naturaleza humana, en cuyo diario vivir y en cualquier actividad genera una cantidad de desechos que se debe considerar (Castillo, 2013).

La basura doméstica es un problema para cualquier administración pública, pues, entre los dañosque desarrolla la sociedad industrializada, uno de los mayores es la proliferación de este tipo deresiduos en las ciudades. El volumen del desecho que debe ser manejado se ha vuelto tan importante,que la extensión de los tiraderos no puede considerarse ya como una solución aceptable. Esteproblema representa un peligro real para la salud de las poblaciones ya que provoca olores, plagasy enfermedades ( Ojeda Benitez, 1998).

El tema de residuos sólidos nace a partir de la Conferencia de Naciones Unidas sobre el Medio Ambiente y Desarrollo en Brasil (1992) (ONU, 1972). Fruto de esta conferencia, está la Agenda 21 que establece las bases para el manejo integral de residuos como parte del desarrollo sostenible, y que reafirma los objetivos que fueron propuestos en la Cumbre Mundial sobre Desarrollo Sostenible (2002) en Johannesburgo, donde se propuso para el 2015, reducir al mínimo los desechos y aumentar, en la medida de lo posible, la reutilización y el reciclaje de materiales, en aras de minimizar los efectos adversos sobre el medio ambiente y mejorar la eficiencia de los recursos.

El manejo de residuos sólidos inicia con la generación y acumulación temporal, continúa con la recolección, transporte y transferencia, y termina con la acumulación final. Un buen manejo de residuos sólidos debe tener como propósito, la reducción de la producción, el reciclaje, la recolección, el tratamiento y la disposición final adecuada. Por el contrario, un manejo inadecuado puede conllevar, en gran medida, a generar problemas de salud pública, pues la velocidad de producción de estos residuos supera los procesos 
de degradación, lo cual incide en el aumento de los focos de contaminación. La deficiente disposición de estos residuos impacta significativamente el paisaje. Por lo tanto, no sólo afecta la salud y el ambiente, sino que disminuye la calidad de vida en términos de espacio y horizonte (Acurio et al., 1998). Para prevenir o mitigar los posibles impactos negativos al ambiente, es imprescindible mejorar su gestión, especialmente la disposición final y el tratamiento de los mismos. El incremento incontrolado de los niveles de consumo y la escasa participación de la población en el manejo de residuos sólidos han presupuesto la urgente búsqueda de soluciones a esta problemática, en la que la educación ambiental juega un papel importante para la adecuada decisión sobre alternativas de solución y exige la toma de conciencia y la contribución de todos y todas para poner en práctica estrategias que busquen mitigar los impactos causados por los residuos dispuestos en el ambiente (Universidad de San Buenaventura, 2012).

A través de estudios bibliográficos y publicaciones en revistas científicas de centros académicos de gran prestigio, se evidencia la problemática ambiental que genera el inadecuado proceso de gestión de desechos sólidos, existen legislaciones concretas para el efecto en cada país del mundo, de América latina y Ecuador, que expiden normas y procedimientos destinados a estandarizar la eliminación de estos residuos que si no son tratados adecuadamente constituyen un problema para la salud y el medio ambiente.

En el Ecuador, lanormativa delMinisterio del Ambiente., Texto Unificado de Legislación Secundaria del Ministerio del Ambiental (TULSMA) Libro VI Anexo 6., Quito-Ecuador., Ministerio del Ambiente., 2006., Pp 429-430, 436-441, 443-448, 452,467 (Ministerio del ambiente, 2006).

La presente norma técnica es dictada bajo el amparo de la Ley de Gestión Ambiental y del Reglamento a la Ley de Gestión Ambiental para la Prevención y Control de la Contaminación Ambiental y se somete a las disposiciones de éstos, es de aplicación obligatoria y rige en todo el territorio nacional.

Esta Norma establece los criterios para el manejo de los desechos sólidos no peligrosos, desde su generación hasta su disposición final. La presente Norma Técnica no regula a los desechos sólidos peligrosos.

La norma tiene como objetivo la Prevención y Control de la Contaminación Ambiental, en lo relativo al recurso aire, agua y suelo.

El objetivo principal de la presente norma es salvaguardar, conservar y preservar la integridad de las personas, de los ecosistemas y sus interrelaciones y del ambiente en general. 
Las acciones tendientes al manejo y disposición final de los desechos sólidos no peligrosos deberán realizarse en los términos de la presente Norma Técnica.

En la provincia de Orellana la gestión de los desechos sólidos se realiza de acuerdo a la;Ordenanza única ambiental., №.303., Orellana-Ecuador., GADMFO., 2012., Pp. 113-121, del Gobierno Autónomo Descentralizado Municipio de Francisco de Orellana (Solvesa, 2017).

Al margen de la legislación ecuatoriana que establece con claridad cuáles son los procesos a ejecutar para la gestión de los desechos sólidos, es común observar que en determinados lugares se cumplen parcialmente las disposiciones ocasionado problemas ambientales y de salud.

La provincia de Orellana cumple parcialmente la norma, se limita a ejecutar procesos de recolección, transporte y disposición final de los residuos generados en la ciudad.

La mayoría de las municipalidades sólo se limitan a cubrir parcial y medianamente los procesos de recolección, transporte y disposición final de los residuos generados en las ciudades, una de estas ciudades es Francisco de Orellana o también conocida como el Coca.

En la parroquia Taracoa, en la provincia de Orellana, en las viviendas es común observar sacos de yute, cartones, fundas plásticas con los desechos sólidos, a la espera del carro recolector, que los transporte, o lo que es peor, abandonados en las aceras y esquinas a la intemperie, constituyendo un medio contamínate para el sector si se considera que perros, rompen las fundas, destruyen los cartones, desgarran los sacos en busca de restos alimenticios y arrastran los desperdicios por toda la comunidad.

La problemática continúa al evidenciarse que no se clasifica la basura como lo determina la "Norma Técnica ecuatoriana INEN 2841, de estandarización de colores para recipientes de depósito y almacenamiento temporal de residuos sólidos", y se dispone para la recolección del carro encargado de hacerlo (Gobierno Autónomo Descentralizado Municipio Francisco de Orellana, 2012).

Con el objetivo de aportar al conocimiento sobre los de residuos que se genera en la parroquia, se realiza un análisis estadístico para conocer los tipos de desechos, cantidades y procedencia en la parroquia de Taracoa Provincia de Orellana.

Desechos Sólidos

\section{DEFINICIÓN}

Conjunto de materiales sólidos de origen orgánico e inorgánico (putrescible o no) que no tienen utilidad práctica para la actividad que lo produce, siendo procedentes de las actividades domésticas, comerciales, industriales y de todo tipo que se produzcan en una comunidad, con la 
excepción de las excretas humana (Gobierno Autónomo Descentralizado Parroaquia Rural de Taracoa).

\section{MATERIALES Y MÉTODOS}

La Parroquia Taracoa forma parte del Cantón Orellana, que está dividido en 11 parroquias rurales y una parroquia urbana, como se puede ver en el mapa de división política del Cantón Orellana, tomado del PDOT actualizado del GADMFO 2014-2019. La parroquia Taracoa se ubica en la zona superior central del Cantón.

La parroquia Taracoa se encuentra localizada en la Provincia de Orellana, Cantón Orellana, al norte de la región amazónica del Ecuador. Geográficamente se encuentra en las coordenadas de latitud Sur $0^{\circ} 28^{\prime} 1,74^{\prime}$ " y $0^{\circ} 35^{\prime} 11,17^{\prime \prime}$ y, entre $76^{\circ} 46^{\prime} 26,24$ " y $76^{\circ} 50^{\prime} 19,07^{\prime \prime}$ de longitud oeste.

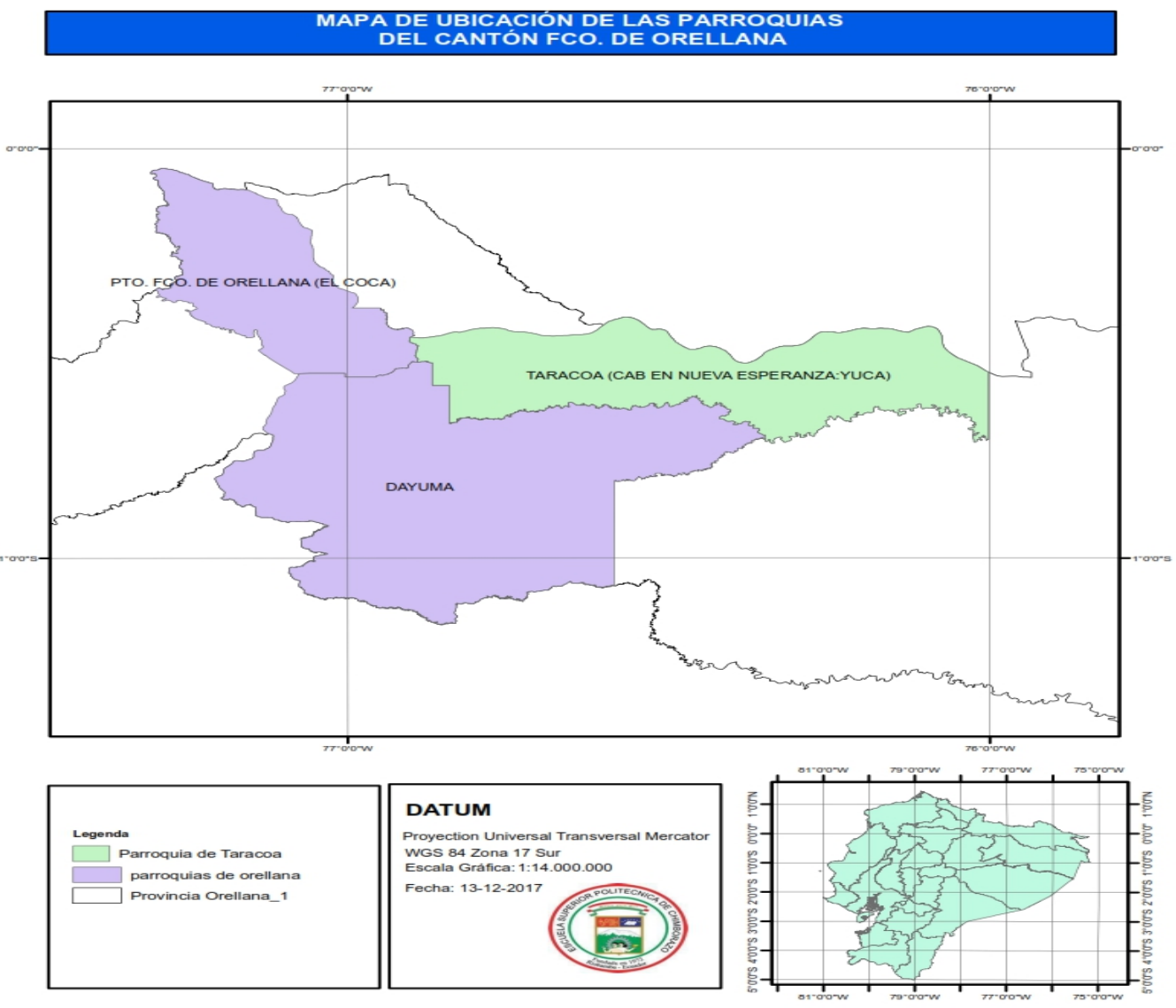

Población. Según datos del CPV 2010, la parroquia tiene una población total de 2616 habitantes, de acuerdo con el siguiente detalle:

Extensión: $411,41 \mathrm{Km} 2$. La densidad bruta de población parroquial es de 6,36 habitantes/km2 al año 2010. De acuerdo con las proyecciones de 
población establecidas por el INEC, el crecimiento poblacional mantendrá una tasa de crecimiento similar y por tanto se espera un aumento proporcional en los valores de densidad bruta.

La red hidrográfica está constituida por los ríos Indillama $(13,4 \mathrm{~km})$; río Curiyacu $(7,74 \mathrm{~km})$; río Rumiyacu $(4,9 \mathrm{~km})$ y otros menores $(154,62 \mathrm{~km})$. El territorio de Taraco está comprendido en la Cuenca del Rio Napo, con una superficie aproximada de $131.72 \mathrm{~km} 2$. Lalaguna Taracoa constituye un recurso hídrico de aguas superficiales con una superficie de $0,38 \mathrm{~km}^{2}$.

La red vial está conformada por tramos asfaltados, lastrados, en arcilla y senderos, según información del PDOT Parroquial anterior. El tramo asfaltado se encuentra solo en la vía principal de comunicación entre la ciudad de El Coca Taracoa, predominando los tramos lastrados inter parroquiales, caminos de herradura y sectores sin acceso vial.

Extensión: 411,41 Km2. La densidad bruta de población parroquial es de 6,36 habitantes $/ \mathrm{km} 2$ al año 2010. De acuerdo con las proyecciones de población establecidas por el INEC, el crecimiento poblacional mantendrá una tasa de crecimiento similar y por tanto se espera un aumento proporcional en los valores de densidad bruta.

\section{Pobreza}

Según la información del PDOT del GAD Municipal de Francisco de Orellana, GADMFO, 2012- 2022, la pobreza por ingreso permite realizar una aproximación a las inequidades y desequilibrios territoriales existentes en el cantón, producto principalmente de "la distribución inequitativa de los ingresos generados por el petróleo en los últimos 30 años". No existe certeza sobre los ingresos promedios de las personas en el Cantón Orellana por lo que basados en el Censo Económico INEC 2010, se estima en 450,65 USD mensuales. Aquellos que laboran en actividades petroleras, minas y canteras tienen un ingreso de 330,76 USD mensuales. Sin embargo, de acuerdo con la encuesta cantonal realizada el año 2011 por el GAD municipal, en el marco de la elaboración del Plan de Igualdad de Oportunidades, PIO, del cantón, el $45,16 \%$ señalaron percibir un ingreso menor a 100 USD mensuales.

En la Parroquia Taracoa se presentan indicadores que evidencian una grave situación de desigualdad y pobreza. En primer lugar, según datos del SIISE 2013, la Incidencia de la Pobreza por NBI tiene una tasa de 96,70\%, frente al $60,1 \%$ de promedio nacional. La incidencia de la Pobreza Extrema por NBI afecta al $49,19 \%$ de la población, muy superior a la media del Ecuador de $26,8 \%$.

Cabe mencionar adicionalmente que destaca la incidencia del trabajo infantil en la parroquia, con una tasa de $8,87 \%$, frente al $6 \%$ a nivel nacional (Taboada-González Paúl et al., 2011). 


\section{Estudio de Caracterización}

La caracterización de los desechos sólidos se expone en la Tabla N. 1. Tabla N. 1. Componentes de los desechos sólidos

\begin{tabular}{|lc|}
\hline COMPONENTE & $\%$ \\
\hline Cartón & $\mathbf{2 , 0 0}$ \\
\hline Varios compuestos & $\mathbf{1 , 3 4}$ \\
\hline Cueros & $\mathbf{2 , 8 2}$ \\
\hline Inorgánicos & $\mathbf{2 , 5 5}$ \\
\hline Madera & $\mathbf{2 , 0 1}$ \\
\hline Metales ferrosos & $\mathbf{3 , 5 5}$ \\
\hline Metales no ferrosos & $\mathbf{0 , 1 3}$ \\
\hline Orgánicos cocina & $\mathbf{3 4 , 1 8}$ \\
\hline Orgánicos jardín & - \\
\hline Papel & $\mathbf{1 0 , 7 2}$ \\
\hline Peligrosos & $\mathbf{0 , 0 7}$ \\
\hline Plásticos alta densidad & $\mathbf{2 , 8 2}$ \\
\hline Plásticos baja densidad & $\mathbf{5 , 7 0}$ \\
\hline Textiles & $\mathbf{4 , 0 2}$ \\
\hline Vidrios & $\mathbf{2 , 0 1}$ \\
\hline Caucho & $\mathbf{1 , 3 4}$ \\
\hline Hueso & $\mathbf{0 , 8 0}$ \\
\hline \hline Menor a 2 cm & $\mathbf{2 4 , 1 3}$ \\
\hline
\end{tabular}

Fuente: "Plan Director de Residuos Sólidos", tomo I, Municipio de Quito pag. 65

Para establecer la generación de los desechos sólidos en la parroquia de Taracoa se utilizó la siguiente metodología, las muestras se tomaron directamente de las viviendas identificadas para el muestreo.

El estudio se efectivizó en coordinación con el departamento de Residuos Sólidos de la Dirección de Higiene y Saneamiento Ambiental del Gobierno Autónomo descentralizado Municipal de Francisco de Orellana.

Se socializó a los moradores de la parroquia Taracoa de las actividades a realizar para determinar para la caracterización de los residuos sólidos y la metodología a utilizar para el efecto.

La parroquia Taracoa cuenta con 162 viviendas, según el censo poblacional del 2010 , con un promedio de 5 habitantes por vivienda, arrojando un total de 817 personas.

Para determinar el número de muestras para la Parroquia Taracoa de utilizó la siguiente fórmula.

$$
n=\frac{z^{2} p q N}{e^{2}(N-1)+z^{2} p q}
$$

\section{Dónde:}

$\mathrm{N}$ : tamaño conocido de la población (500)

e: error máximo permitido $(10 \%)$ 
z: Margen de confiabilidad constante 1,96

p: probabilidad que el evento ocurra $(0.5)$

q: probabilidad que el evento ocurra $(0.5)$

n: tamaño de la muestra.

$\mathrm{n}=60.50$

$$
\begin{gathered}
n=\frac{(1.96)^{2}(0.25) 162}{(0.1)^{2}(162-1)+(1.96)^{2}(0.25)} \\
n=\frac{155,58}{1,61+0,96}
\end{gathered}
$$

$\mathrm{n}=61$

Una vez obtenida la muestra, se identificaron los sitios de muestreo.

Para determinar la Producción Per- cápita de la Parroquia Taracoa perteneciente al Cantón Puerto Francisco de Orellana, Provincia, se realizó el muestreo por un periodo de quince días, se procedió a pesar el volumen de los desechos eliminados. fórmula:

Para determinar la producción per cápita se utilizó la siguiente

$$
P P C=\frac{\text { peso de la basura }\left(\begin{array}{c}
\mathrm{Kg} \\
\text { dia }
\end{array}\right)}{\text { Numero de habitantes }(\mathrm{hab})}
$$

$$
\begin{gathered}
P P C=\frac{273,60}{305} \\
P P C=0,89
\end{gathered}
$$

Para establecer la caracterización de los desechos sólidos de la parroquia Taracoa, se procedió a recolectar los desechos sólidos directamente de los domicilios, trasladados a un centro de acopio donde se pesaron y clasificaron los mismos.

\section{RESULTADOS}

De las 61 familias escogidas para realizar el estudio, con un promedio de 5 habitantes por cada una de ellas da un total de 305 habitantes, los resultados se evidencian en la Tabla N. 2.

Tabla N.2. Producción per cápita

\begin{tabular}{|l|c|}
\hline FAMILIAS & 61 \\
\hline TOTAL, HABITANTES DEL SECTOR & 305 \\
\hline PRODUCCIÓN DIARIA & $273,60 \mathrm{~kg}$ \\
\hline PPC & $0,89 \mathrm{~kg}$ \\
\hline DENSIDAD & 300 \\
\hline
\end{tabular}


Figura N. 1. Producción per cápita

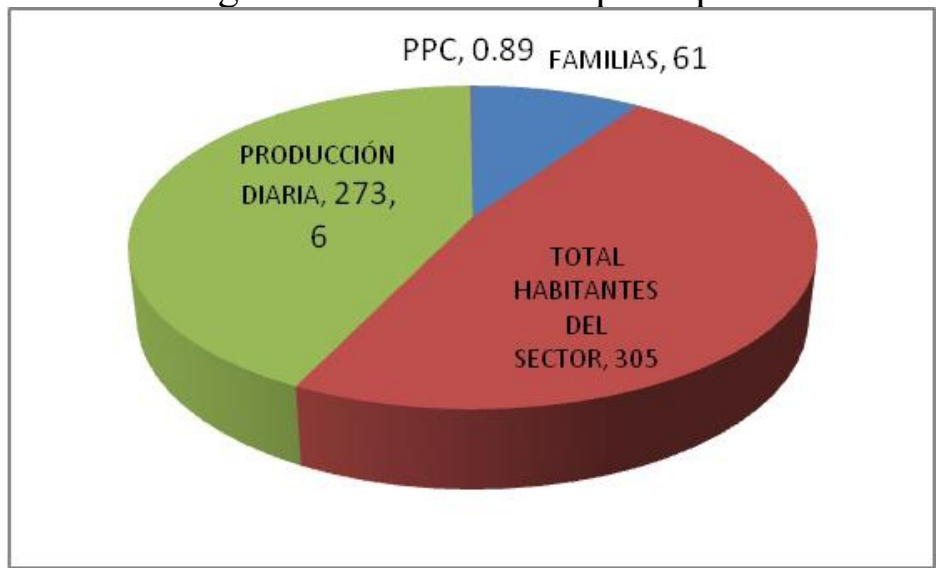

La PPC de desechos sólidos en la parroquia Taracoa de la provincia de Orellana fue de 0,89 kg/hab/día, arrojando una producción diaria de 273,60 $\mathrm{kg} /$ día.

\section{Caracterización de los Desechos Sólidos}

Referente a la caracterización de los residuos sólidos los resultados se evidencian en la Tabla N. 3.

Tabla N. 3. Caracterización de desechos sólidos

\begin{tabular}{|l|c|}
\hline SUBPRODUCTOS & $\mathbf{~ K g}$ \\
\hline Orgánico & 163,71 \\
\hline Plástico & 11,26 \\
\hline Cartón & 10,61 \\
\hline Papel & 8,55 \\
\hline Latas & 3,78 \\
\hline Fundas plásticas & 18,62 \\
\hline TOTAL & $\mathbf{2 1 6 , 5 5}$ \\
\hline
\end{tabular}

Los desechos orgánicos en los domicilios son los de mayor peso referente a los demás sub productos, seguido de las fundas plásticas, siendo el de menor volumen las latas en las que se incluyen atún y refrescos.

Una vez clasificados los desechos por productos, se pesan por separado, para obtener el peso en fracción de cada uno de ellos, se utiliza la siguiente ecuación:

Dónde:

$$
P \%=\frac{G 1}{G} * 100
$$

$\mathrm{P} \%=$ porcentaje del subproducto

G1=peso del subproducto

$\mathrm{G}=$ peso total de los subproductos.

Nota 
Tabla N. 4. Sub productos

\begin{tabular}{|l|c|}
\hline SUBPRODUCTOS & $\mathbf{\%}$ \\
\hline Orgánico & 75,60 \\
\hline Botellas de plástico & 5,20 \\
\hline Cartón & 4,90 \\
\hline Papel & 3,95 \\
\hline Latas & 1,75 \\
\hline Fundas plásticas & 8,60 \\
\hline TOTAL & $\mathbf{1 0 0} \%$ \\
\hline
\end{tabular}

\section{DISCUSIÓN}

Según; el Programa 'PNGIDS' del Ministerio del Ambiente del Ecuador; actualmente la generación de residuos en el país es de 4,06 millones de toneladas métricas al año y una generación per cápita de $0,74 \mathrm{~kg} .10$. La PPC de residuos sólidos en la parroquia de Taracoa de la provincia de Orellana de $0,89 \mathrm{~kg}$, lo que se encuentra sobre la referencia del Ministerio del Ambiente del país, este aumento tiene se explicación en los hábitos de la población que teniendo limitadas condiciones de centros de acopio donde adquirir sus productos en la parroquia, acuden a la cabecera cantonal Francisco de Orellana (el Coca), para abastecerse de productos, lo que hace que se consuma un exceso de fundas plásticas para transportar los productos (PNGIDS, 2017).

Para la caracterización de residuos sólidos nos basamos en el estudio de residuos sólidos identificados en los trabajos de Estrada y Tejeda (2009:813) y que sustentanel estudio de mérito, corresponden a las siguientes categorías: a) materia orgánica (restos dealimentos de origen animal y/o vegetal); b) plástico (bolsas, envoltorios, envases de agua y de gaseosa,vasos y platos de poliestireno, desechos de PVC (recipientes de alimentos y restos de tuberías);c) papel y cartón (cajas, propaganda, periódicos y revistas); d) vidrio (botellas de bebidas, vasos yplatos); e) metal ferroso (latería de alimentos y pinturas, alambres de construcción, clavos); f) metalno ferroso (latas de aluminio, residuos de material eléctrico); g) madera (cajones tablas y ramas); h)pañales y trapos; i) contaminantes químicos: (pilas, baterías, medicamentos, lámparas fluorescentes); j) contaminantes biológicos (papel higiénico, toallas sanitarias, hisopos, algodón, vendas, preservativos) (Estrada \& Tejeda, 2009).

Referente a los porcentajes los desechos orgánicos en los domicilios son los de mayor porcentaje con el 75,60\%, seguido de las fundas plásticas con el 8,60\%, los botellas de plástico con el 5,20\%, el cartón con el 4,90\%, el papel con el 3,95\% y latas con el $1,75 \%$.

\section{CONCLUSION}

En el estudio realizado se pudo evidenciar que los residuos sólidos no son clasificados adecuadamente, los habitantes de la parroquia mesclan los 
desechos y así los disponen en las veredas para que sean recopilados por los carros recolectores, la producción per cápita es $0,89 \mathrm{Kg} / \mathrm{hab} /$ día, generando un total de $216,55 \mathrm{~kg} /$ día, los cuales son enviados al botadero del Municipio de Orellana.

El análisis estadístico determina que en los domicilios de la parroquia Taracoa de la Provincia de Orellana los desechos orgánicos ocupan el primer lugar con el 75,60 \%, lo cual permite establecer que este tipo de desecho es el que se genera en mayor grado en la comunidad en estudio.

Debido a la inexistencia de estudios que arrojen datos sobre la estadística de los residuos sólidos en el sector, no se pueden establecer comparaciones de los datos generados, por lo que este estudio servirá de línea de base futuros estudios en el Oriente ecuatoriano.

\section{References:}

1. Castillo, L. (2013). Evaluación del manejo de residuos sólidos en la Universidad Pontificia Bolivariana seccional Bucaramanga. Rev Fac Ing UPTC; 22:71-84.

2. Estrada Vázquez, C. \& Tejeda Rosas, M. (2009). Estudio de generación de residuos sólidos no domiciliarios (Servicios) para el proyecto Relocalización y diseño del relleno sanitario del Municipio de San Pedro Mixtepec, Juquila, Oaxaca. Coordinación de Promoción del Desarrollo. México.

3. Gobierno Autónomo Descentralizado Municipio Francisco de Orellana (2012). Ordenanza única ambiental., Nº303.

4. Gobierno Autónomo Descentralizado Parroaquia Rural de Taracoa. Actualización del Plan de Ordenamiento Territorial Taracoa.

5. Ministerio del ambiente (2006). Texto Unificado de Legislación Secundaria del Ministerio del Ambiental (TULSMA). Quito.

6. Ojeda Benitez, S. (1998). Análisis estadístico del comportamiento de los residuos sólidos domiciliarios en una comunidad urbana. Front Norte; 10.

7. ONU (1972). Informe de la Conferencia de las Naciones Unidas sobre el Medio Humano [Internet]. Estocolmo; Available from: http://www.un.org/spanish/esa/sustdev/agenda21/riodeclaration.htm

8. PNGIDS (2017). Programa Nacional para la Gestión Integral de Desechos Sólidos - PNGIDS ECUADOR [Internet]. [cited Nov 16]. Available from: http://www.ambiente.gob.ec/programa-pngidsecuador/

9. Solvesa (2017). Plan de manejo de desechos sólidos en la Gestión Ambiental [Internet]. [cited Nov 12]. Available from: http://www.solvesacorp.com/solvesacorp.com/docs/downloads/Plan de manejo de desechos solidos en la Gestion Ambiental.pdf 
10. Taboada-González Paúl, A., Aguilar-Virgen Quetzalli, \& OjedaBenitez, S. (2011). Avances en Ciencias e Ingeniería. Rev Ing.;2.

11. Universidad de San Buenaventura (2012). Diagnóstico preliminar, base para la construcción de un Programa de Manejo de residuos Sólidos. Gestión y Ambient.;18. 